\section{$\underset{\substack{\text { hommes } \\ \text { \& migrations }}}{ }$}

\section{Hommes \& migrations}

Revue française de référence sur les dynamiques

migratoires

1316 | 2017

L'islam en Europe

\title{
De l'intégration des élèves immigrés à celle des élèves musulmans en Suisse
}

Une analyse à partir de deux faits divers médiatisés

\section{Geneviève Mottet}

\section{(2) OpenEdition}

Journals

Édition électronique

URL : http://journals.openedition.org/hommesmigrations/3800

DOI : $10.4000 /$ hommesmigrations.3800

ISSN : 2262-3353

Éditeur

Musée national de l'histoire de l'immigration

Édition imprimée

Date de publication : 1 mars 2017

Pagination : 89-97

ISBN : 978-2-919040-37-7

ISSN : 1142-852X

Référence électronique

Geneviève Mottet, « De l'intégration des élèves immigrés à celle des élèves musulmans en Suisse », Hommes \& migrations [En ligne], 1316 | 2017, mis en ligne le 01 mars 2020, consulté le 10 décembre 2020. URL : http://journals.openedition.org/hommesmigrations/3800 ; DOI : https://doi.org/10.4000/ hommesmigrations.3800 


\title{
DE L'INTÉGRATION DES ÉLĖVES IMMIGRÉS À CELLE DES ÉLĖVES MUSULMANS EN SUISSE
}

\section{UNE ANALYSE À PARTIR DE DEUX FAITS DIVERS MÉDIATISÉS}

Par GENEVIEVVE MOTTET, docteure en sciences de l'éducation,

Faculté de psychologie et des sciences de l'éducation (FPSE), université de Genève.

\begin{abstract}
Si les faits divers médiatiques cristallisent les passions de la société civile, ils sont également, parfois, le vecteur de changements de politiques publiques. L'analyse de deux événements en milieu scolaire en Suisse, en 1999 et 2016, montre leur contribution à la focalisation des débats autour de l'intégration des élèves immigrés et de la place de l'islam à l'école et, plus largement, dans la société helvète. Dans un contexte de tensions identitaires exacerbées, l'instrumentalisation des faits divers à des fins politiques révèle des mécanismes de stigmatisation des musulmans pour les faire correspondre aux attentes de la société dominante.
\end{abstract}

Après un demi-siècle de présence des élèves issus de la migration dans les discours des médias, les images qui les concernent ont changé en substance comme en intensité au fil du temps. À partir de la seconde moitié des années 1990, les articles traitant des jeunes issus de l'immigration apparaissent et deviennent de plus en plus fréquents dans la presse, se concentrant toujours plus autour des jeunes musulmans. Certains faits divers, transformés en affaires souvent très médiatisées, participent de la production sociale de nouveaux stigmates. Cet article présente deux faits divers, survenus en Suisse, qui ont eu un impact sur les politiques éducatives, sur l'intégration des familles issues de la migration, ainsi que sur la manière dont la question musulmane est appréhendée 
dans les discours publics. Cette dernière n'apparaît qu'en filigrane en 1999 lors du premier fait divers, alors qu'elle est au centre du second, en 2016. Ces deux faits divers représentent deux situations distinctes. D'une part, un drame : le meurtre d'un enseignant par le père d'un élève kosovar à SaintGall, en 1999. D'autre part, l'affaire de l'école de Therwil : le «scandale " des

A l'instar du contexte

politique français et européen décrit par Éric

Fassin et Didier Fassin, la Suisse est également en train d'investir la question raciale,

la question religieuse et la thématique de l'islam. deux élèves musulmans qui refusent de serrer la main de leurs professeures et le tollé contre une direction qui le leur permet, en 2016. Nous tâcherons de comprendre la transformation de ces faits divers en faits de sociétés et la façon dont leurs traitements ont eu une influence directe sur l'accélération du développement d'une politique d'intégration globale en direction des familles immigrées d'abord, puis des populations de religion musulmane.

\section{Comprendre la construction des représentations négatives des immigrés}

Différents travaux académiques, des ouvrages et de nombreux articles ont montré le traitement négatif progressif de la question de l'immigration dans le discours politico-médiatique et comment, au tournant des années 1980, la presse de droite comme de gauche est passée d'un cadrage sur les problèmes vécus par les immigrés (logement, droits, alphabétisation) aux problèmes posés à la
France par les immigrés (délinquance, baisse de niveau dans les écoles, terrorisme, etc.) ou encore par les problèmes d'intégration ${ }^{1}$.

Robert Castel relevait que les principaux épisodes de la montée en puissance de l'islamophobie ont été la première guerre du Golfe en 1991², à laquelle ont succédé le déclenchement de la seconde Intifada palestinienne en 2000 , les attentats du 11 septembre 2001, la guerre en Irak, puis d'autres attentats en Europe, etc. Le sociologue soulignait l'impact de ces événements à la fois sur les jeunes que leur origine rattache à l'islam et sur l'opinion publique. Depuis lors, nous assistons à de nouveaux attentats qui alimentent le discours sur la menace islamiste jusque dans l'enceinte scolaire à partir de thèmes comme la violence, l'égalité des sexes ou la laïcité. À l'instar du contexte politique français et européen décrit par Éric Fassin et Didier Fassin ${ }^{3}$, la Suisse est également en train d'investir la question raciale, la question religieuse et la thématique de l'islam

Des chercheurs montrent les enjeux de la montée en force de la laïcité dans un contexte de "panique morale ${ }^{5}$ ». De même, Amel Boubekeur parle d' « islam de crise $^{6} »$. Pour lui, les débats sur le rôle de l'islam dans ces crises sont le plus souvent présentés en termes d'inadaptabilité culturelle pouvant mener au "clash des civilisations", tels que l'ont été les questions du port du voile, des émeutes en banlieue et l'affaire des caricatures islamophobes. Il s'agit bien d'interroger comment ces questions morales, qui émergent des faits divers, participent à évincer l'ethnicisation des rapports sociaux et l'exclusion des populations allogènes ${ }^{7}$, ainsi que le déficit de citoyenneté que

1. Simone Bonnafous, «La médiatisation de la question immigrée : état des recherches. Études de communication. Techniques d'expression, information, communication ", in La médiatisation des problèmes publics, vol. 22, 1999, pp. 59-72 ; Gérard Noiriel, Immigration, antisémitisme et racisme en France (XIXe-XXe siècle). Discours publics, humiliations privées, Paris, Fayard, 2007 ; Yvan Gastaut, «1983, tournant médiatique de l'immigration en France », in Hommes \& migrations, n 1313, 2016, pp. 6-9. 2. Robert Castel, La discrimination négative. Citoyens ou indigènes?, Paris, Seuil, 2007. 3. Didier Fassin, Éric Fassin (dir.), De la question sociale à la question raciale, Paris, La Découverte, 2006. 4. Gaëtan Clavien, « Médias et discours islamophobes : au croisement du dicible et du recevable ", in Mallory Schneuwly Purdie, Matteo Gianni, Magali Jenny (dir.), Musulmans d'aujourd'hui : identités plurielles en Suisse, Genève, Labor et Fides, 2009, pp. 95-110. 5. Sylvain Crépon, « La lutte pour la reconnaissance des signes religieux à l'école. Une étude comparative France-Belgique ", in Politique européenne, vol. 1, n² 24, 2008, pp. 83-101; Geneviève Zoïa, “Morale laïque et identité à l'école ", in Le Télémaque, vol. 1, n 43, 2013, pp. 73-86 ; François Durpaire, Béatrice Mabilon-Bonfils, Fatima moins bien notée que Marianne, La Tour-d’Aigues, L'Aube, 2016 ; Pierre Merle, « Faut-il refonder la laïcité scolaire? ", in La Vie des idées, 17 février 2015. 6. Amel Boubekeur, « L'européanisation de l'islam de crise », in Confluences Méditerranée, vol. 2, n 57, 2006, pp. 9-23. 7. Simona Tersigni, " "Prendre le foulard”: les logiques antagoniques de la revendication ", in Mouvements, vol. 5, $\mathrm{n}^{\circ}$ 30, 2003, 116-122. 
subissent les jeunes de banlieue ${ }^{8}$. Aussi, loin de légitimer les actes violents et les événements dramatiques, il s'agit, à la suite de Béatrice MabilonBonfils ${ }^{9}$, d'explorer les soubassements dans notre imaginaire collectif de la tendance à analyser le monde à partir des catégories de la menace.

Cet article s'appuie sur des sources récoltées dans le cadre d'une enquête longitudinale qui investit l'analyse des processus de diffusion, de mise en circulation et de légitimation d'une "rhétorique de la science de la diversité ${ }^{10} »$ dans le champ scolaire ${ }^{11}$. Alors que nous avons effectué une analyse longitudinale des articles parus dans un quotidien genevois, la Tribune de Genève ${ }^{12}$, entre les années 1970 à 2015, nous intéressant autant à ceux concernant la question scolaire qu'à ceux traitant de la question migratoire, nous avons observé l'émergence d'un ciblage sur le problème, en Suisse, des jeunes d'origine musulmane dans la seconde moitié des années 1990, au moment de la montée en force de l'extrême droite (parti de l'UDC ${ }^{13}$ ) qui deviendra le second parti du pays en 1999.

Les sources mobilisées dans cet article ont été choisies parce qu'elles illustrent l'impact des faits divers sur le processus de problématisation des élèves immigrés, catégorisés comme musulmans. De même, parce qu'elles renseignent sur la manière dont des acteurs multipositionnés transforment et font circuler les catégories de perception des immigrés et de l'islam dans le champ scolaire. On s'appuiera sur la lecture de plus de 100 articles de presse, d'une quinzaine de rapports de recherche et de mandats institutionnels de diverses institutions (Forum suisse pour les migrations, ministère national de l'Éducation ${ }^{14}$, Commission fédérale des étrangers, etc.), d'une dizaine de documents politiques (rapports, mémoriaux, textes de loi) qui se réfèrent explicitement aux deux faits divers que nous avons présentés (ou qui en découlent en partie), ainsi que de textes qui informent sur la création de mesures promouvant la laïcité (associations, brochure sur la laïcité, etc.).

Nous verrons comment ces formes de problématisations ont des impacts concrets sur la relation sociale des élèves et de leur famille subissant ces présupposés et sur la mise en place de dispositifs potentiellement stigmatisants et contraignant les concernant (élèves et parents). Nous ver- Un fait divers ayant eu lieu rons également dans quels en 1999 à Saint-Gall a été contextes émergent chacun convoquéà de nombreuses de ces faits divers, et com- reprises dans les sources ment les mobilisations qui étudiées. Il s'agit du meurtre en découlent sont ancrées d'un enseignant parle père dans une logique proche d'un élève kosovar. de l'islamophobie portée à l'échelle mondiale sachant, comme le souligne Patrick Haenni ${ }^{15}$, que c'est dans les années 1990 que la Suisse découvre «sa » minorité musulmane et, ce faisant, commence à penser et à définir son rapport à elle. Il relève que l'on assiste depuis lors à une « internalisation » progressive de la question de l'islam et des musulmans en Suisse.

\section{Un fait divers comme accélérateur des politiques d'intégration}

Un fait divers ayant eu lieu en 1999 à Saint-Gall a été convoqué à de nombreuses reprises dans les sources étudiées. Il s'agit du meurtre d'un enseignant par le père d'un élève kosovar. Les articles de presse, ainsi que les références à ce fait divers par de nombreux acteurs mentionnent régulièrement la nationalité du père, soit son origine koso-

8. Robert Castel, «La discrimination négative. Le déficit de citoyeneté des jeunes de banlieue ", in Annales. Histoire, sciences sociales, $n^{\circ}$ 4, 2006, pp. 777-808. 9. Béatrice Mabilon-Bonfils, Geneviève Zoïa, La laïcité au risque de l'autre, La Tour d'Aigues, éd. de l'Aube, 2015 ; Béatrice Mabilon-Bonfils, "L'école après Charlie », in Le sujet dans la cité, vol. 2, n 6, 2015, pp. 37-47. 10. Joseph Gusfield, La culture des problèmes publics. L'alcool au volant: la production d'un ordre symbolique, Paris, Economica, 1981. 11. Geneviève Mottet, À l'"École de la diversité". Enquête sur la fabrique d'une politique éducative, Thèse de doctorat en sciences de l'éducation, Genève, Université de Genève, 2013. 12. Nous avons également récoltés un certain nombre d'articles provenant d'autres quotidiens romands. 13. Union démocratique du centre (UDC), parti conservateur, libéral, souverainiste. 14. En Suisse, l'organe correspondant est la CDIP (Conférence suisse des directeurs cantonaux de l'instruction publique). 15. Patrick Haenni, "Dynamiques sociales et rapport à l'État. L'institutionnalisation de l'Islam en Suisse ", in Revue européenne de migrations internationales, vol. 10, nº 1, 1994, pp. 183-198. 
vare. Connaissant le contexte politique de la fin des années 2000 (arrivée de nombreux réfugiés d'ex-Yougoslavie, difficultés économiques, montée de l'extrême droite) $)^{16}$, on peut considérer que l'usage de ce fait divers a servi à mettre au cœur de la politique d'intégration la question des familles immigrées dans le champ scolaire.

Plus que la cause de l'acte, l'origine étrangère (kosovare) du père de l'élève

La mise en place de mesures

de soutien pour les est mise en exergue dans les articles. Le Temps, dans son titre relatif à cette affaire : enseignants est également réclamée suite au drame.

Des structures et des

dispositifs visant à promouvoir l'intégration des étrangers sont petit à petit créés dans les cantons

qui institutionnalisent

des bureaux d'intégration

et créent des « contrats

d'intégration ».

avec lequel il serait en interdépendance, était légitime. La Commission fédérale des étrangers $\left(\mathrm{CFE}^{18}\right)$, ainsi que le Forum suisse pour l'étude des migrations ${ }^{19}$ se réfèrent aussi à cet événement et relèvent qu'il a participé au débat sur l'intégration des immigrés. Selon la CFE, «le cas tragique du meurtre de Spirig [...] a éveillé la conscience de toute la Suisse, faisant de l'intégration un thème central pour de nombreuses années ${ }^{20} »$. Différents thèmes seront développés par des acteurs de tous bords suite à cet événement. Les débats portent sur le taux d'étrangers dans les classes, sur les écoles multiculturelles, sur la question du quota d'élèves étrangers dans les classes, ainsi que sur les classes séparées demandées par certains. La mise en place de mesures de soutien pour les enseignants est également réclamée suite au drame. Des structures et des dispositifs visant à promouvoir l'intégration des étrangers sont petit à petit créés dans les cantons qui institutionnalisent des bureaux d'intégration et créent des « contrats d'intégration ». On observe le développement de discours exigeant des étrangers qu'ils prouvent leur bonne volonté de s'intégrer, notamment par l'apprentissage de la langue d'accueil. En cas de manques de volonté à ce niveau ou en cas de comportement délinquant des parents ou des enfants, il est question de retirer le permis de séjour attribué par la Confédération. De même, des amendes sont données en cas d'absence des parents aux réunions d'école ou en cas d'absence des enfants à l'école. Le slogan « encourager et exiger » est prôné, attendant des familles étrangères qu'elles s'intègrent à la société d'accueil de manière inédite.

\section{La récupération politique des dispositifs publics destinés aux immigrés}

Bien sûr, tous ces dispositifs et discours ne sont pas issus de ce seul « fait divers ». Certains étaient déjà en discussion dans le contexte politique et économique que nous avons évoqué. Toutefois, il est intéressant de souligner que celui-ci a été mentionné par des acteurs politiques de tous bords, ainsi que par des chercheurs du ministère national de l'Éducation, du Forum suisse des migrations ou de la Commission fédérale des étrangers, etc. En outre, les références explicites à cet événement se poursuivent au moins jusqu'en 2008. En effet, dans l'état des lieux d'un catalogue de mesures du Parti radical genevois (parti de droite) intitulé «Une nouvelle dynamique pour Genève » paru en

16. Il est dit dans un article que nous avons recensé que le père d'élève kosovar était catholique (Le Temps, 14 janvier 1999). Cependant, à cette exception près, les articles que nous avons recensés ne se réfèrent pas à la religion du père. Sachant qu'au Kosovo, l'islam représente la religion de plus de $90 \%$ de la population, les lecteurs sont portés à croire que le meurtrier est musulman. 17. Le Temps, 13 janvier 1999. 18. CFE, L'intégration des migrantes et des migrants en Suisse. Faits. Secteurs d'activités. Postulats, Berne, Commission fédérale des étrangers, 1999, p. 16. 19. Sandro Cattacin, Kaya Bülent, Le développement des mesures d'intégration de la population migrante sur le plan local en Suisse, Neuchâtel, Forum suisse pour l'étude des migrations, 2001. 20. CFE, L'intégration des migrantes et des migrants en Suisse, op. cit., p. 37. 
février 2008, ce parti rappelle justement l'affaire de Saint-Gall qui « avait défrayé la chronique » en janvier 1999, concernant l'assassinat d'un enseignant par le père d'un élève. Cet état des lieux s'inquiète des cas de violence dans le milieu scolaire et se réfère aux problèmes liés à l'intégration de populations migrantes ${ }^{21}$. Une des mesures demandées par le parti vise à faire suivre aux enseignants une formation sur la gestion des différences culturelles et religieuses. Les mesures proposées sont proches de celles qui ont été prises par le canton de Saint-Gall suite à ces événements ${ }^{22}$. Il est intéressant d'observer que les mesures proposées par le Parti radical genevois, déposées en février 2008, s'inscrivent directement dans le prolongement de celles de l'UDC en août $2007^{23}$. Si l'on s'intéresse aux propositions de l'UDC dans leur document : " Pour l'ordre et la sécurité. Halte à la violence juvénile et à la criminalité étrangère ${ }^{24}$ ", il s'agit d'apporter des modifications au droit pénal des mineurs et au droit civil, en augmentant la responsabilité des parents et en amendant ces derniers en cas d'écarts à la loi de la part de leurs enfants. La tendance générale semble être à la mise en place de mesures de plus en plus sévères visant à imposer l'ordre et la sécurité tout en "punissant les parents non responsables ».

Si ce fait divers ne s'accompagne que d'une problématisation de la question religieuse des personnes d'origine étrangère en filigrane, il a fonctionné comme un catalyseur du manque d'intégration des familles immigrées dans le débat public. Le second fait divers que cet article présente s'inscrit quant à lui au cœur de la question religieuse. Il participe à problématiser l'islam de manière inédite dans le champ éducatif. Les modalités initiées petit à petit par les politiques d'intégration (menaces relatives au permis d'établissement, amendes, encouragement à l'intégration, etc.) depuis le début des années 2000 seront également mobilisées. En outre, une batterie de nouvelles mesures verra aussi le jour.

\section{Un fait divers comme catalyseur de la question musulmane à l'école}

Le fait divers de la " poignée de main » a eu lieu en avril 2016, soit dix-sept ans après le premier. Le traitement qui lui est fait révèle, selon nous, la montée en puissance d'une nouvelle forme de problématisation plus large de l'intégration des élèves immigrés qui cible spécifiquement les élèves musulmans. Nous avons également choisi de présenter ce fait divers parce qu'il est survenu récemment et que l'emploi qui en est fait doit être compris à la lumière du contexte politique mondial de lutte contre les islamistes intégristes. Bien sûr, des années 2000 à aujourd'hui, d'autres événements ont été mobilisés par les médias et les politiques, abordant la thématique de l'intégration des élèves immigrés et de leurs parents : événements médiatisés sur la « violence » de jeunes étrangers, le port du voile, l'exemption des cours de natation ou le Si ce fait divers ne s'accompagne que d'une problématisation de la question religieuse des personnes d'origine étrangère en filigrane, il a fonctionné comme un catalyseur du manque d'intégration des familles immigrées dans le débat public burkini.

Les premiers articles relatant le fait divers informent que la direction d'un établissement du canton de Bâle-Campagne a dispensé deux élèves de saluer leurs professeures par une poignée de main. Le problème n'a pas été énoncé publiquement par la direction d'établissement mais par d'autres acteurs, notamment par l'intermédiaire du journal Schweiz am Sonntag. Selon Swissinfo du 6 avril 2016, cette poignée de main "agite toute la Suisse ": "Ces derniers jours la Suisse a vécu un emballement médiatique sur le thème de la poignée de main qui s'est propagé sur les réseaux sociaux 
et même jusqu'à l'étranger. En cause : deux élèves syriens dispensés par la direction de l'école de serrer la main à leur institutrice pour des motifs religieux ${ }^{25}$. " Selon la Tribune de Genève du 7 avril $2016^{26}$, le « compromis » trouvé par la direction de l'établissement a été de demander aux élèves de saluer verbalement leurs professeurs (femmes et hommes). Il remonte au mois de novembre précédent. La dérogation, une fois médiatisée, a soulevé un vent de réactions, quasi unanimes, pour condamner une mesure

Le titre d'un article de la Tribune de Genève

du 12 avril 2016 monte

l'affaire en épingle en liant les deux jeunes musulmans

à Daech : «Un des frères relaie la propagande de

Daech ». Dès lors, les journalistes enquêtent sur

les origines de la famille des deux frères. On apprend que celle-ci ne serait pas « intégrée » à la société suisse. considérée comme discriminatoire envers les femmes et portant atteinte aux « valeurs suisses ». Le quotidien genevois relève la réaction de la conseillère fédérale socialiste, Simonetta Sommaruga, suite à la dénonciation médiatique de cet événement : « $\mathrm{Ce}$ n'est pas ainsi que je conçois l'intégration. " Le problème dénoncé amène rapidement une vive polémique qui obligera la hiérarchie à revenir sur sa décision, notamment suite à la demande du ministère national de l'Éducation (CDIP). Des acteurs hétérogènes et de tous bords (responsables des politiques éducatives, syndicats d'enseignants, députés, etc.) s'expriment sur l'événement dans les médias des différents cantons et critiquent bien souvent la décision de l'établissement en question, invoquant des thèmes comme l'égalité hommes-femmes, la laïcité, la citoyenneté, etc. Face à l'inexistence de directive du ministère cantonal de l'Éducation $\left(\mathrm{DIP}^{27}\right)$ relative à ce genre de situation, une expertise juridique a dû être commanditée pour savoir s'il est légalement permis de demander à la direction d'établissement de modifier sa décision.
La presse relève que cette affaire a créé une « polémique nationale » comme en attestent les intitulés : "Accepter que des musulmans ne serrent pas la main de leur maitresse, une décision inadmissible et hypocrite (Le Temps, 6 avril 2016), «La poignée de main relance la question d'imams suisses ${ }^{28}$ " (Tribune de Genève, 8 avril 2016) ; "L'affaire des deux ados syriens, dispensés de serrer des mains, s'était jusqu'ici cantonnée à un débat de société» (20 minutes, 12 avril 2016); ; L'école secondaire de Therwil (BL) avait provoqué un tremblement de terre en Suisse en avril » (20 minutes, 25 mai 2016). Des mesures sont réclamées par divers acteurs qui s'expriment sur le sujet. Le PS (Parti socialiste) de Bâle-Campagne demande que des mesures soient prises par le canton pour éviter et sanctionner ce genre de comportement contre les femmes. Le PDC (Parti démocrate-chrétien ${ }^{29}$ annonce la préparation d'une intervention au Grand Conseil. L'UDC, quant à elle, informe du dépôt d'une motion visant à inscrire dans la loi l'interdiction d'exceptions pour les communautés religieuses.

\section{La construction médiatique de la menace de la radicalisation à l'école}

La majorité des points de vue présentés par la presse dénoncent la décision prise par la direction d'établissement. De même, lors d'une émission «Infrarouge » de la RTS (Radio télévision de Suisse romande) du 18 avril 2016 portant sur l'événement et intitulée "Quelle place pour l'islam en Suisse ", les invités sont très critiques et l'imam invité est le seul à interroger la réalité de ce problème de la poignée de main. Il est passablement rudoyé par différents intervenants. L'analyse de cette table ronde va dans le sens des constats de Pierre Tévanian ${ }^{30}$ qui observe que,

25. Sibilla Bondolfi, «Débat sur l'intégration des musulmans. Une poignée de main qui agite toute la Suisse », in Swissinfo.ch., 6 avril 2016. 26. Gabriel Sassoon, "Rien n’arrête la polémique sur la poignée de main ", in Tribune de Genève, 7 avril 2016. 27. Il s'agit du Département de l'instruction publique (DIP). 28. Mesgarzadeh et al. constatent que la construction d'un islam de Suisse est en marche. Voir Samina Mesgarzadeh, Sophie Nedjar, Mounia Bennani-Chraïbi, "L'"organisation” des musulmans de Suisse. Dynamiques endogènes et injonctions de la société majoritaire ", in Elisa Banfi, Christophe Monnot (dir.), La Suisse des mosquées : derrière le voile de l'unité musulmane, Genève, Labor et Fides, 2013, pp. 53-76. 29. Parti politique suisse situé au centre de l'échiquier politique. 
lors des débats médiatiques portant sur l'interdiction du voile à l'école, la majorité des interventions soutiennent l'interdiction alors que celles qui s'y opposent sont souvent acculées voire interrompues dans leurs interventions et les filles touchées par cette interdiction totalement réduites au silence et ignorées.

Le titre d'un article de la Tribune de Genève du 12 avril 2016 monte l'affaire en épingle en liant les deux jeunes musulmans à Daech : "Un des frères relaie la propagande de Daech ». Dès lors, les journalistes enquêtent sur les origines de la famille des deux frères. On apprend que celle-ci ne serait pas "intégrée " à la société suisse. On informe que le père est imam à la mosquée du roi Faysal à Bâle, mosquée réputée pour être radicale. À partir de là, la menace du radicalisme islamiste dans le domaine des politiques d'immigration rejoint la question scolaire. Il est proposé de développer la formation d'imams en Suisse et d'interdire le financement des mosquées par des fonds en provenance de l'étranger ${ }^{31}$. En raison de l'affaire, la procédure de naturalisation demandée par la famille a été ajournée (24 heures, 18 avril), soit juste deux semaines après la mise en avant de l'affaire par la presse : «Après le tollé médiatique provoqué par ce refus, l'Office cantonal de la migration a convoqué la famille pour discuter. » On peut faire ici le parallèle avec l'une des mesures proposées suite au premier fait divers, mesure mettant en question la prolongation du permis d'établissement des parents de jeunes «délinquants».

Fin mai, le ministère de l'Éducation de BâleCampagne annonce officiellement la fin de la dispense pour les deux élèves musulmans (20 minutes, 25 mai 2016). Le vide législatif est levé pour le ministère de l'éducation de ce canton, puisque l'analyse juridique effectuée relève que "[l]'intérêt public concernant l'égalité entre femme et homme aussi bien que l'intégration de personnes étrangères l'emportent largement sur la liberté de croyance des élèves " (24 heures, 25 mai 2016). Diverses mesures sont alors prises pour répondre à ce type de situations. Par exemple, il est officiellement mentionné que, selon la législation, les parents ou les responsables légaux risquent un avertissement, voire une amende pouvant aller jusqu'à 5000 francs. D'autres mesures disciplinaires envers les élèves sont également proposées. Selon le quotidien 20 minutes du 25 mai 2016, le DIP (ministère de l'Éducation) a annoncé vouloir créer les bases juridiques nécessaires afin d'établir " une passerelle entre la loi sur l'éducation et celle sur les étrangers». Il est également dit que "[l]es écoles sont tenues de signaler à l'office des migrations des problèmes substantiels liés à l'intégration ". Le quotidien romand 20 minutes du 19 septembre 2016 informe du développement du projet de loi qui sera soumis à consultation en novembre, attendant des directions d'école qu'elles signalent à l'Office de la migration les cas de refus de serrer la main d'un enseignant.

À côté de ces réponses sécuritaires liées au traitement de ce fait divers, les thèmes de la laïcité sont au cœur des débats politiques en Suisse. Par exemple, actuellement à Genève, trois projets de loi sur la laïcité ont été déposés (entre 2015 et 2016), le A côté de ces réponses sécuritaires liées au traitement de ce fait divers, les thèmes de la laïcité sont au cœur des débats politiques en Suisse. Par exemple, actuellement à Genève, trois projets de loi sur la laïcité ont été déposés (entre 2015 et 2016), le « réseau laïque romand » a été créé (2016), I'UDC a lancé une initiative anti-burqa (2016). " réseau laïque romand " a été créé (2016), l'UDC a lancé une initiative anti-burqa (2016). Dans le contexte scolaire, les enseignants genevois ont reçu une brochure intitulée «La laïcité à l'école » (rentrée 2016), ce qui représente un fait inédit. Dans le canton du 
Valais, une initiative contre le voile à l'école a été déposée par l'UDC qui a obtenu le nombre de signatures requis pour demander au gouvernement cantonal de légiférer

Si les politiques éducatives cherchent néanmoins à rassurer sur l'ampleur numérique du problème

(peu de situations) et à promouvoir le dialogue avec

les élèves et les parents

musulmans, elles veulent

également envoyer un message clair sur les limites institutionnelles données à la liberté de religion. en la matière. On assiste également à des débats contre la création de jardins d'enfants et d'écoles enfantines islamiques dans certains cantons. La multiplicité des actions et des acteurs engagés dans la " cause laïque » et la montée en puissance des débats publics sur l'islam et l'école, depuis le tournant des années 2010 en Suisse, doivent être interrogés quant à leurs réelles capacités à développer le "vivre ensemble " et la " cohésion sociale " prônés en filigrane.

\section{Conclusion}

Tout comme Julien Beaugé et Abdellali Hajjat l'ont observé pour la France ${ }^{32}$, la construction du «problème musulman » en Suisse comprend des problématisations successives de l'islam et s'accompagne du développement du « champ de la laïcité » et de la " pédagogie de la laïcité ». Avec la sur-politisation et la sur-médiatisation de la question musulmane relativement au contexte scolaire, ainsi que la mobilisation d'acteurs hétérogènes qui s'investissent dans la résolution du problème de l'intégration des élèves musulmans, nous assistons bien à ce qu'Herbert Blumer $^{33}$ nomme la construction d'un problème public.

Alors que le premier fait divers, au tournant des années 2000, semble être un tremplin pour le déploiement d'une politique d'intégration
" sociale sécuritaire » des étrangers où se dessine en filigrane une problématisation de l'élève d'origine musulmane, le traitement du second fait divers montre que la question musulmane est aujourd'hui explicitement au cœur de la politique d'intégration prônée. Si les politiques éducatives cherchent néanmoins à rassurer sur l'ampleur numérique du problème (peu de situations) et à promouvoir le dialogue avec les élèves et les parents musulmans, elles veulent également envoyer un message clair sur les limites institutionnelles données à la liberté de religion. Elles créent en ce sens des directives pour informer les professionnels de l'éducation des attitudes à avoir en cas de comportement d'élèves menaçant le maintien de la laïcité à l'école et promeuvent une "pédagogie de la laïcité » pour "maintenir » la cohésion sociale. Évoquant les questions d'intégration des minorités ethniques en France et en Grande-Bretagne, Adrian Favell ${ }^{34}$ remarque que les responsables politiques "dépendent de plus en plus de prises de position dogmatiques sur les valeurs, les vertus et les idéaux nationaux sacrés ». Khadija Mohsen-Finan et Christophe Bertossi montrent en ce sens comment le débat qui se focalise sur l'islam permet d'envisager la réélection et le soutien populaire, dans un contexte où l'identité nationale est définie à la fois par le «haut » (l'Europe) et par le «bas » (les identités particulières) $)^{35}$.

Tout se passe comme si le traitement du fait divers de «la poignée de main » traduisait l'idée d'une adaptation nécessaire des élèves d'origine musulmane aux normes et aux valeurs locales, soit à la production de musulmans de Suisse modérés dans leur rapport à l'islam. La solution proposée par les politiques éducatives vise en ce sens au maintien des élèves risquant de se radicaliser dans une identité religieuse mesurée. Un rapport de l'Office fédéral des migrations ${ }^{36}$ insiste d'ailleurs sur le rôle actif que devraient 
jouer les musulmans modérés en se mettant en réseau pour faire entendre leurs voix en diffusant "des informations "positives" " sur leurs pratiques dans l'objectif de conserver la "paix sociale ». Samina Mesgarzadeh et al. relèvent ainsi que la construction progressive d'une identité de "musulmans de Suisse » ou de "musulmans modérés " se fait en réaction à une stigmatisation qui pourrait amener certains groupes à se radicaliser ${ }^{37}$.

Alors que l'on encourage les pratiques modérées des populations musulmanes dans et hors le champ sco- laire, il s'agit d'interroger à quel point les politiques en Suisse se dessaisissent elles-mêmes paradoxalement d'un discours mesuré relativement à la laïcité pour se focaliser sur cette question de manière fortement avivée. Ainsi, le travail de mobilisation autour de l'intégration des élèves musulmans (et de leur famille) élude le fait que, dans la très grande majorité des cas, ceux-ci adhèrent aux principes de la citoyenneté. Khadija Mohsen-Finan et Christophe Bertossi relèvent en ce sens que les recherches sociologiques basées sur des terrains empiriques ne cessent de faire le même constat ${ }^{38}$. 